Highly Energetic Physical Processes and

Mechanisms for Emission from Astrophysical Plasmas

IAU Symposium, Vol. 195, 2000

P. C. H. Martens, S. Tsuruta, and M. A. Weber, eds.

\title{
Jets from Black Hole Magnetospheres
}

K. Shibata

Kwasan Observatory, Kyoto University, Yamashina, Kyoto 607-8471, Japan

S. Koide

Faculty of Engineering, Toyama University, Toyama 930-8555, Japan

T. Kudoh

National Astronomical Observatory, Mitaka, Tokyo 181-8588, Japan

S. Aoki

Dept. of Astronomy, Univ. Tokyo, Bunkyo-ku, Tokyo 113-0033, Japan

\begin{abstract}
Recent general-relativistic MHD simulations of jets ejected from black-hole magnetospheres (for both Schwarzschild and Kerr holes) have revealed that (1) strong shock waves are formed in the accretion flow inside $3 r_{s}$, (2) jets show two-layered shell structure consisting of a gas-pressure driven jet and a magnetically driven jet, the former being accelerated from a high-pressure region heated by strong shocks, and (3) in the case of a Kerr hole, magnetically driven jets are produced from the ergosphere by the effect of frame dragging.
\end{abstract}

\section{Introduction}

Magnetically driven jets from accretion disks are one of the most promising models for astrophysical jets, such as AGN jets (active galactic nuclei: e.g., see Lovelace 1976; Blandford 1976; Blandford \& Payne 1982) and YSO jets (young stellar objects: e.g., see Pudritz \& Norman 1986; Uchida \& Shibata 1985). The merit of this model is that it can naturally explain both acceleration and collimation of jets by magnetic force in helically twisted magnetic flux tubes created by the rotation of the disk. This model explains also the extraction of angular momentum from an accretion disk, enabling efficient accretion of disk plasma onto central objects. Although there are still controversial arguments about collimation (e.g., Spruit 2000), this model has been extensively studied in a framework of steady jet models. Recent observations, however, revealed various evidences of time variation in jets. Thus, the need for nonsteady jet models is now increasing rapidly.

The first nonsteady MHD simulations of magnetically driven jets from accretion disks have been carried out by a Japanese group (Shibata \& Uchida 

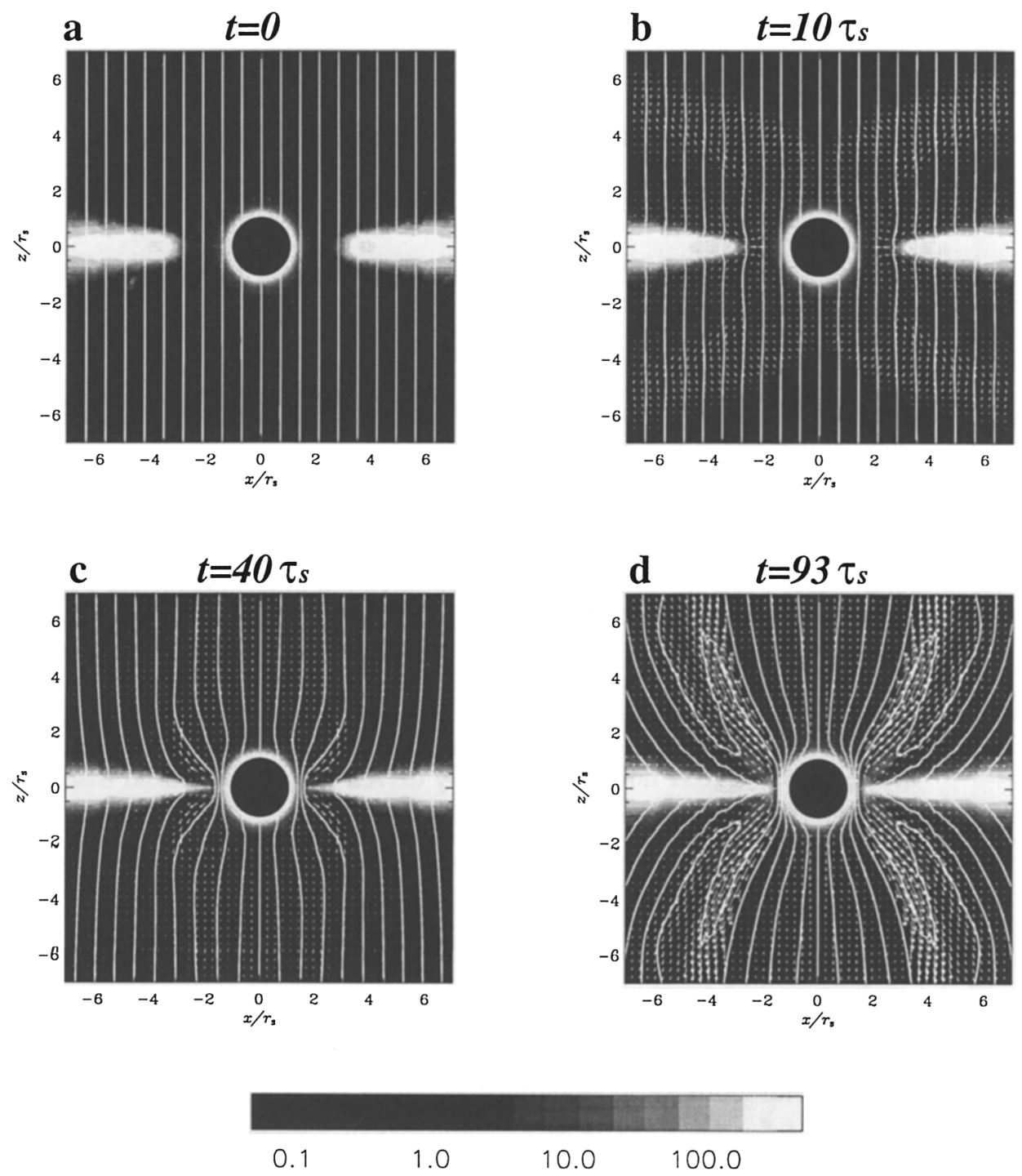

Figure 1. Evolution of the jet formation in a Schwarzschild blackhole magnetosphere. The black circle indicates a black hole (i.e., the region inside the Schwarzschild radius, $r<r_{s}$ ). The solid lines are magnetic field lines, the vectors are velocity vectors, and the gray scale shows the proper-mass density in a logarithmic scale. The distance and time are in units of $r_{s}$ and $\tau_{s}=r_{s} / c$, where $c$ is the speed of light. 
1985, 1986; Uchida \& Shibata 1985). Since then, this kind of nonsteady simulation (including the accretion disk self-consistently) has been extended to various cases, such as a non-point-mass gravitational potential (Shibata \& Uchida 1987), collision of MHD jets with interstellar dense clouds (Shibata \& Uchida 1990), thick disks (Matsumoto et al. 1996; Kudoh et al. 1998), Balbus-Hawley unstable disks (Stone \& Norman 1994; Kato et al. 2000), three dimensions (Matsumoto $\&$ Shibata 1996), and even the cases with magnetic reconnection (Hayashi et al. 1996; Hirose et al. 1997). These simulations showed that the velocity of MHD jets is of the order of the Keplerian speed at the foot point of the jets (with weak dependence on magnetic field strength), and this empirical rule has been nicely explained by Kudoh \& Shibata $(1995,1997)$ using 1D, steady, semi-analytical models.

All these simulations were based on nonrelativistic models. However, AGN jets and microquasar jets are believed to emanate from the close vicinity of black holes at the center of these objects since the jets often show superluminal motion, suggesting that these jets are relativistic; the Lorentz factor of jets is about 10 for AGN jets (e.g., 3C273, Pearson et al. 1981) and $\sim 2$ for Galactic microquasars (Mirabel \& Rodriguez 1994). There is also evidence of black holes in AGN (e.g., see Tanaka et al. 1995, Miyoshi et al. 1995). Hence, it is necessary to develop a general-relativistic MHD jet model to explain these jets.

Koide, Shibata, \& Kudoh $(1998 ; 1999)$ performed nonsteady, general-relativistic (GR) MHD simulations of jets ejected from black-hole accretion disks for the first time. Aoki et al. (2000) examined in detail the dependence of this GRMHD jet model on the density of the disk. More recently, Koide et al. (2000) extended the model to that for a Kerr hole. Hereafter, we will summarize the main findings by these recent GRMHD simulations of jets.

\section{Method of GRMHD Simulations}

We use $3+1$ formalism (Thorne, Price, \& Macdonald 1986) to solve generalrelativistic (GR) MHD equations; time and spatial derivatives are separated, and we use physical quantities relative to a ZAMO (zero angular momentum observer). For simulations on a Kerr hole, see Koide et al. (2000).

Initially, we assume a Keplerian cool (dense) disk penetrated by a uniform magnetic field with a nonrotating, hydrostatic, isothermal corona (Koide et al. 1998) or a freely falling corona (Koide et al. 1999) outside of the disk. The inner edge of the disk is located at the last stable orbit, $3 r_{s}$, where $r_{s}$ is the Schwarzschild radius. The basic parameters of this problem are

$$
\begin{gathered}
E_{m g}=\frac{\text { magnetic energy }}{\text { gravitational energy }}=\left(\frac{V_{A}}{V_{k}}\right)^{2} \sim 10^{-3}, \\
E_{t h}=\frac{\text { thermal energy }}{\text { gravitational energy }}=\left(\frac{C_{s}}{V_{k}}\right)^{2} \sim 10^{-3}, \text { and } \\
\frac{\rho_{d}}{\rho_{c}}=\frac{\text { density of disk }}{\text { density of corona }} \sim 40-400 .
\end{gathered}
$$

A simplified TVD scheme (Davis 1984; Koide et al. 1999) has been used to solve the GRMHD equations. A special-relativistic MHD version of the code 


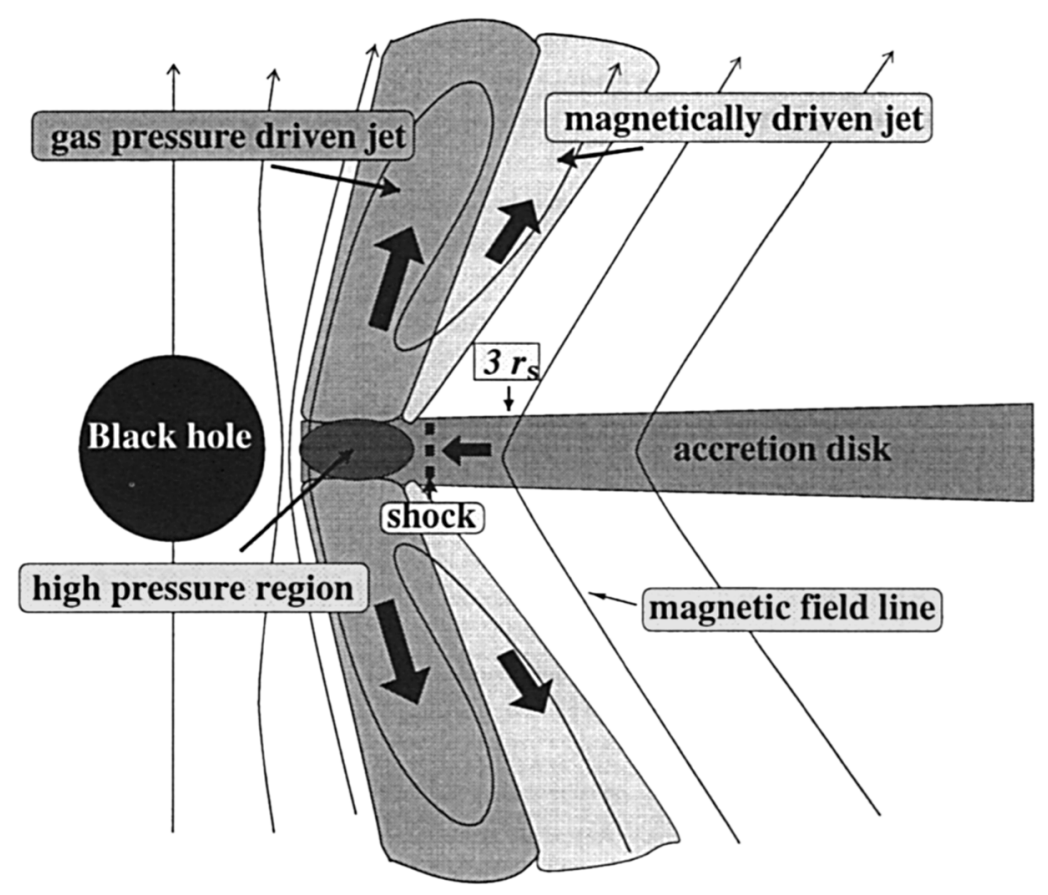

Figure 2. Schematic picture of the jet formation from the accretion disk around the black hole. Below the last stable orbit of Keplerian motion $r=3 r_{s}$, the accretion disk falls into the hole rapidly. The shock is formed in the rapidly falling disk. The gas-pressure driven jet is formed by the extremely high pressure behind the shock front, and the magnetically driven jet is formed in the outer layer of the gaspressure driven jet.

has been developed by Koide, Nishikawa, \& Mutel (1996). We assume free boundaries at the surface of the black hole and at the outer boundary. The Regge-Wheeler tortoise coordinate is used, and the number of grid points in a typical case are $210 \times 70$.

\section{Jets from Disks Around a Schwarzschild Hole}

Figure 1 shows a typical example of general-relativistic MHD simulations of jets from an accretion disk around a Schwarzschild black hole. These figures show the rest-mass density (gray scale), velocity (vector), and magnetic field (solid lines) for $-7 r_{s}<x<7 r_{s}$ and $-7 r_{s}<z<7 r_{s}$. At $t=10 \tau_{s}\left(=r_{s} / c, c\right.$ is the speed of light), we can see a front of propagating (torsional) Alfvén waves which is generated by the interaction between the rotating disk and a uniform vertical 
magnetic field. The disk begins to fall through magnetic braking (i.e., angular momentum transport by the Alfvén waves). At $t=40 \tau_{s}$, a jet starts to be ejected from inside $3 r_{s}$, and develops to a hollow, cylindrical jet with maximum speed $\sim 0.88 c$ at $t=93 \tau_{s}$.

Careful examination of this result reveals that the jet has a two-layered shell structure consisting of a fast gas-pressure driven jet in the inner part and a slow magnetically driven jet in the outer part, both of which are collimated by the global poloidal magnetic field penetrating the disk (see Figure 2 for a schematic illustration of the jet formation process). It has also been revealed that $a$ shock wave is produced in the accretion flow inside $3 r_{s}$ because the velocity of accretion flow inside $3 r_{s}$ exceeds the sound speed by the general-relativistic effect (i.e., no stable orbit inside $3 r_{s}$ ). It is this shock wave that enhances gas pressure near the inner edge of the disk and eventually drives the gas-pressure driven jet.

Figure 3 shows a close-up of the jet-forming region near the black hole at $t=91 \tau_{s}$. It is remarkable that both accretion flow and outflow (jets) coexist inside $3 r_{s}$, and the shock wave is generated at $1.8-1.9 r_{s}$. Note that if we assume the same parameters $E_{t h}$ and $E_{m g}$ as those for these relativistic models, we cannot get the strong shock wave and the gas-pressure driven jet in the nonrelativistic MHD models. In this sense, these are characteristic features of general-relativistic MHD jet models. (This does not mean that the shock and the gas-pressure driven jet cannot be formed in Newtonian models. If we assume small initial rotation velocity and low temperature, we can get a shock in a supersonic accretion flow with a gas-pressure driven jet. )

Similar results (shock formation and two-layered shell structure of jets) are also found in the case of a freely falling corona (Koide et al. 1999). The generation of shocks in the accretion flow inside $3 r_{s}$ may be related to the origin of the iron line observed by ASCA (Tanaka et al. 1995).

Aoki et al. (2000) found that the velocity of gas-pressure driven jets increases with increasing density of the disk: $\gamma_{j}{ }^{2}-\gamma_{j} \propto\left(\rho_{d} / \rho_{c}\right)^{0.75}$, where $\gamma_{j}$ is the Lorentz factor of the jets. If we apply this result to Galactic microquasars $\left(\gamma_{j} \sim 2\right)$ and AGN jets $\left(\gamma_{j} \sim 10\right)$, we can predict $\rho_{d} / \rho_{c} \sim 3 \times 10^{3}$ for microquasars and $\rho_{d} / \rho_{c} \sim 10^{5}$ for AGN jets.

\section{Jets from Disks Around a Kerr Hole}

Recently, Koide et al. (2000) have developed a new general-relativistic MHD code in Kerr geometry and have succeeded in simulating MHD jets from a Kerr hole magnetosphere. Figure 4 shows two typical examples of MHD jets in two cases, in which the disk counterrotates (a) and corotates (b) with respect to the black hole's rotation. These figures show the rest-mass density (gray scale), velocity (vectors), and magnetic field (solid lines) for $0<R<7 r_{s}$ and $0<z<7 r_{s}$ at $t=47 \tau_{s}$. The angular momentum parameter of the black hole is $a=0.95$, and the radius is $r_{H}=0.656 r_{s}$. The initial state in the simulation is similar to that for a Schwarzschild hole, though the coronal plasma is assumed to be in a state of nearly stationary infall, with the specific enthalpy $h / \rho c^{2}=1+\Gamma p /\left[(\Gamma-1) \rho c^{2}\right]=1.3$, where $\Gamma=5 / 3$ is the specific heat ratio. Far from the hole, it becomes the stationary transsonic solution exactly. The initial velocity of the accretion disk is assumed to be the Keplerian velocity. 


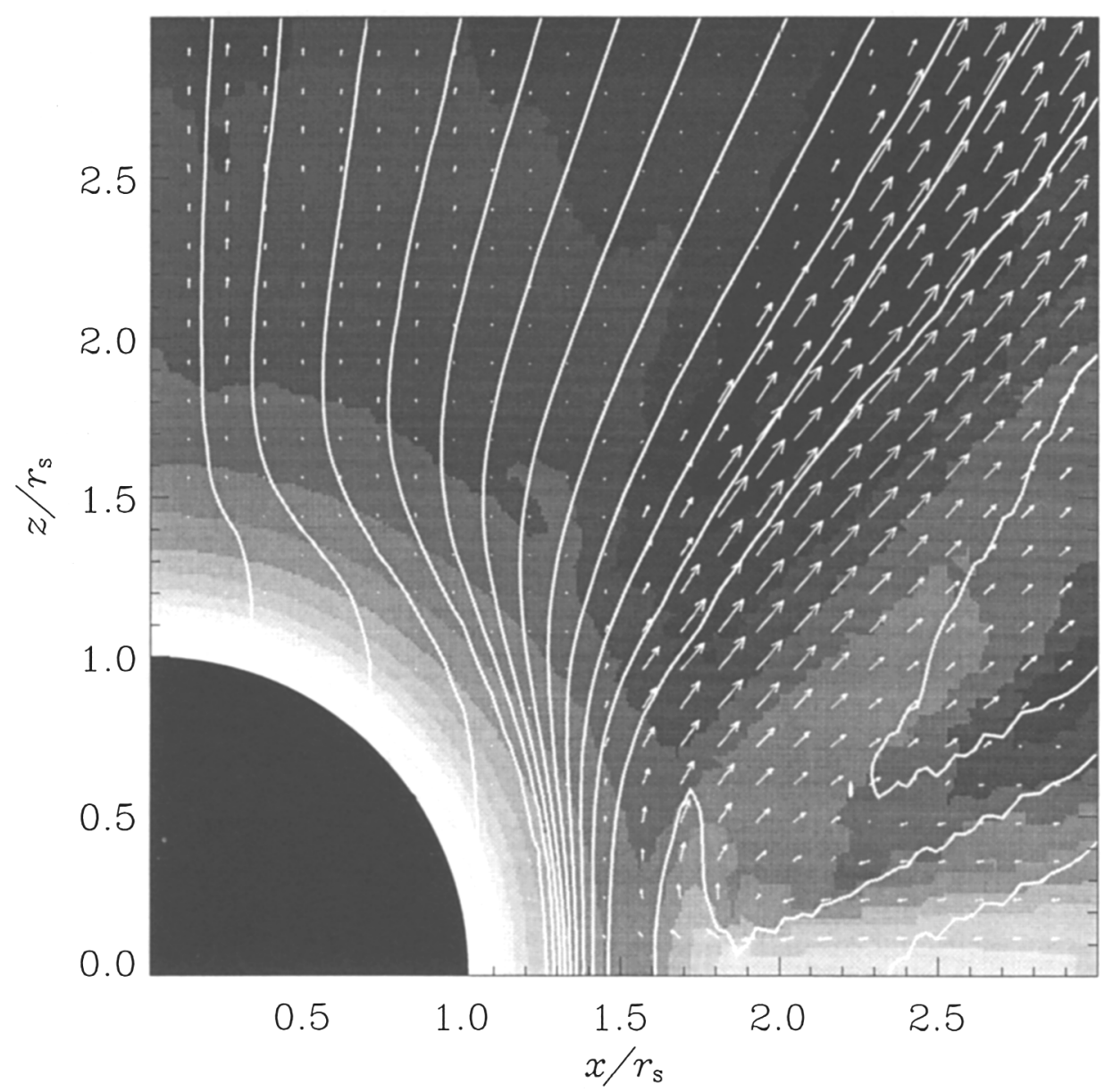

Figure 3. Close-up of the jet production region near a Schwarzschild black hole (at $t=91 \tau_{s}$ in the case shown in Figure 1); gray scale shows the proper-mass density. We can see both outflow and inflow of the disk plasma. 
(a) Counter-rotating disk

$$
\mathrm{t}=47
$$

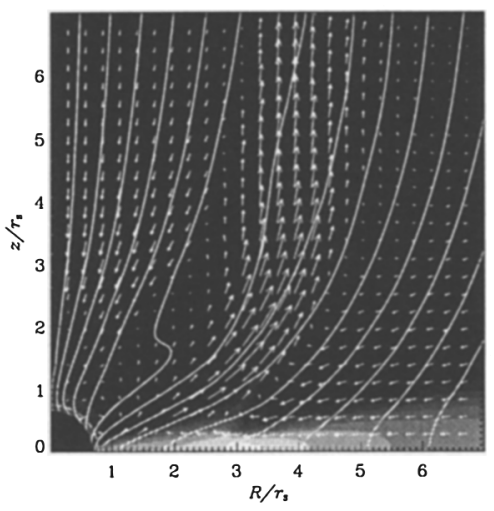

(b) Co-rotating disk

$$
\mathbf{t}=47
$$

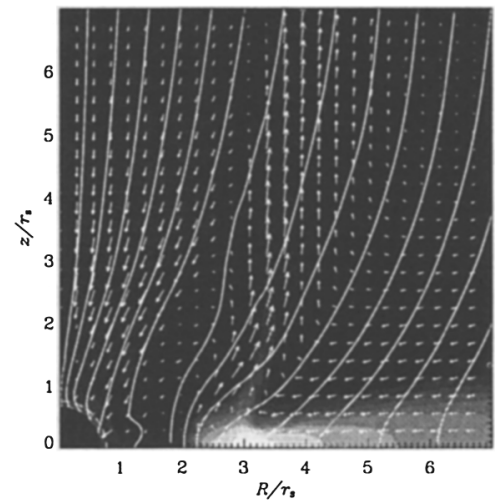

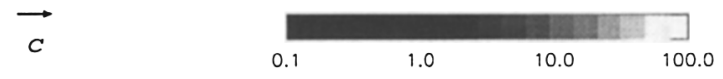

Figure 4. Typical examples of the jet ejected from an accretion disk around a Kerr hole; (a) counterrotating case, (b) corotating case. Note that in the counterrotating case, the disk plasma can fall into the Kerr hole, which enables the formation of a magnetically driven jet from the ergosphere, while, in the corotating case, the disk plasma cannot reach the ergosphere, so there is no ergosphere driven jet.

The corotating disk is stable, but the counterrotating disk is unstable in the region $R<4.4 r_{s}$. Except for the disk rotation direction, we use the same initial conditions in both cases. Because of this difference in stability of a circular orbit in counterrotating and corotating disks, a big difference appears in the jet dynamics. In the counterrotating case, the disk plasma can reach the black hole horizon and ergosphere, so that the magnetic field is strongly twisted by the frame dragging effect, thus generating a magnetically driven jet from the ergosphere. On the other hand, in the corotating disk, the disk cannot reach the black hole horizon, and hence there is no ergosphere driven MHD jet but there are a strong, gas-pressure driven jet and a weak, magnetically driven jet ejected from the accretion disk, as in the case of a Schwarzschild hole. The maximum velocity of these jets is about $0.3-0.4 c$.

\section{References}

Aoki, S. I., Koide, S., Kudoh, T., \& Shibata, K. 2000, these Proceedings Blandford, R. D. 1976, MNRAS, 176, 465

Blandford, R. D., \& Payne, D. G. 1982, MNRAS, 199, 883

Davis, S. F. 1984, NASA Contractor Rep. 172373, ICASE Rep. No. 84-20

Hayashi, M. R., Shibata, K., \& Matsumoto, R. 1996, ApJ, 468, L37

Hirose, S., Uchida, Y., Shibata, K., \& Matsumoto, R. 1997, PASJ, 49, 193 
Kato, S. X., Kudoh, T., \& Shibata, K. 2000, these Proceedings

Koide, S., Nishikawa, K.-I., \& Mutel, R. L. 1996, ApJ, 463, L71

Koide, S., Shibata, K., \& Kudoh, T. 1998, ApJ, 495, L63

Koide, S., Shibata, K., \& Kudoh, T. 1999, ApJ, 522, 727

Koide, S., Meier, D. L., Kudoh, T., \& Shibata, K., 2000, ApJ, in press

Kudoh, T., \& Shibata, K. 1995, ApJ, 452, L41

Kudoh, T., \& Shibata, K. 1997, ApJ, 474, 362

Kudoh, T., Matsumoto, R., \& Shibata, K. 1998, ApJ, 508, 186

Lovelace, R. V. E. 1976, Nature, 262, 649

Matsumoto, R., Uchida, Y., Hirose, S., Shibata, K., Hayashi, M. R., Ferrari, A., Bodo, G., \& Norman, C. 1996, ApJ, 461, 115

Matsumoto, R., \& Shibata, K. 1997, in IAU Colloq. 163, Accretion Phenomena and Related Outflows, eds. D. T. Wickramasinghe et al., PASP Conf. Ser., 121, 443

Mirabel, I. F., \& Rodriguez, L. F. 1994, Nature, 371, 46

Miyoshi, M., et al. 1995, Nature, 373, 127

Pearson, J. J., et al. 1981, Nature, 290, 365

Pudritz, R. E., \& Norman, C. 1986, ApJ, 301, 571

Shibata, K., \& Uchida, K. 1985, PASJ, 37, 31

Shibata, K., \& Uchida, K. 1986, PASJ, 38, 631

Shibata, K., \& Uchida, K. 1987, PASJ, 39, 559

Shibata, K., \& Uchida, K. 1990, PASJ, 42, 39

Stone, J. M. \& Norman, M. 1994, ApJ, 433, 746

Spruit, H. C. 2000, these Proceedings

Tanaka, Y., et al. 1995, Nature, 352, 659

Thorne, K. S., Price, R. H., \& Macdonald, D. A. 1986, Membrane Paradigm (New Haven and London: Yale University Press)

Uchida, Y., \& Shibata, K. 1985, PASJ, 37, 515 